\title{
Seronegative antiphospholipid syndrome: refining the value of "non-criteria" antibodies for diagnosis and clinical management
}

Haematologica 2020

Volume 105(3):562-572

\section{Correspondence: \\ PASQUALE PIGNATELLI \\ pasquale.pignatelli@uniroma1.it. \\ Received: June 10, 2019. \\ Accepted: December 18, 2019. \\ Pre-published: January 30, 2020.}

doi:10.3324/haematol.2019.221945

Check the online version for the most updated information on this article, online supplements, and information on authorship \& disclosures: www.haematologica.org/content/105/3/562

\section{(C)2020 Ferrata Storti Foundation}

Material published in Haematologica is covered by copyright. All rights are reserved to the Ferrata Storti Foundation. Use of published material is allowed under the following terms and conditions:

https://creativecommons.org/licenses/by-nc/4.0/legalcode. Copies of published material are allowed for personal or internal use. Sharing published material for non-commercial purposes is subject to the following conditions:

https://creativecommons.org/licenses/by-nc/4.0/legalcode, sect. 3. Reproducing and sharing published material for commercial purposes is not allowed without permission in writing from the publisher.

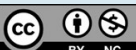

\author{
Pasquale Pignatelli, ${ }^{1,2^{*}}$ Evaristo Ettorre, ${ }^{3 *}$ Danilo Menichelli, ${ }^{1}$ Arianna Pani, ${ }^{4,5}$ \\ Francesco Violi, ${ }^{12^{\star \star}}$ and Daniele Pastori ${ }^{1 * \star}$ \\ ${ }^{1}$ Clinica Medica, Department of Internal Medicine and Medical Specialties, Sapienza \\ University of Rome, Rome; ${ }^{2}$ Mediterranea Cardiocentro, Naples; ${ }^{3}$ Department of \\ Cardiovascular, Respiratory, Nephrologic, Anesthesiologic and Geriatric Sciences, Division \\ of Gerontology, Sapienza University, Rome; ${ }^{4}$ Department of Oncology and Onco- \\ Hematology, University of Milan, Milan and ${ }^{5} \mathrm{Clinical}$ Pharmacology Unit, ASST Grande \\ Ospedale Metropolitano Niguarda, Milan, Italy \\ ${ }^{\star} P P$ and $E E$ contributed equally to this work. \\ $\star * F V$ and DP contributed equally to this work as co-senior authors.
}

\section{ABSTRACT}

A ntiphospholipid syndrome (APS) is a systemic autoimmune disease characterized by arterial and venous thrombotic manifestations and/or pregnancy-related complications in patients with persistently high antiphospholipid antibodies (aPL), the most common being represented by anticardiolipin antibodies (aCL), anti-beta 2 glycoprotein-I (aß2GPI), and lupus anticoagulant (LAC). A growing number of studies have showed that, in some cases, patients may present with clinical features of APS but with temporary positive or persistently negative titers of aPL. For these patients, the definition of seronegative APS (SN-APS) has been proposed. Nevertheless, the negativity to classic aPL criteria does not imply that other antibodies may be present or involved in the onset of thrombosis. The diagnosis of SN-APS is usually made by exclusion, but its recognition is important to adopt the most appropriate anti-thrombotic strategy to reduce the rate of recurrences. This research is in continuous development as the clinical relevance of these antibodies is far from being completely clarified. The most studied antibodies are those against phosphatidylethanolamine, phosphatidic acid, phosphatidylserine, phosphatidylinositol, vimentin/cardiolipin complex, and annexin A5. Moreover, the assays to measure the levels of these antibodies have not yet been standardized. In this review, we will summarize the evidence on the most studied non-criteria aPL, their potential clinical relevance, and the antithrombotic therapeutic strategies available in the setting of APS and SN-APS.

\section{Introduction}

The prevalence of antiphospholipid antibodies (aPL) in the general population is difficult to estimate due to the lack of population-based studies. The most frequently detectable aPL are anticardiolipin antibodies (aCL), anti $\beta 2$-glycoprotein I

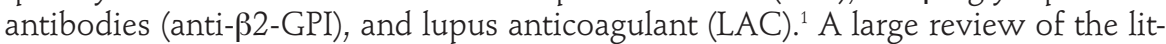
erature in 2013 estimated that the prevalence of aPL positivity is $6 \%$ among women with pregnancy complications, $10 \%$ among patients with deep venous thrombosis (DVT), 11\% among patients with myocardial infarction, and 17\% among patients with juvenile stroke ( $<50$ years of age). As acknowledged by the Authors, this prevalence should be considered with caution, because $60 \%$ of the papers were published before 2000 , all three criteria aPL tests were performed in only $11 \%$ of the papers, and $36 \%$ of papers used a low-titer aCL cut off. ${ }^{2}$

Subjects carrying aPL who develop thrombotic complications are diagnosed with the antiphospholipid syndrome (APS), which was first described in 1983 by Hughes, who initially defined it as "anticardiolipin syndrome". ${ }^{3}$ This definition was derived from clinical observation of recurrent miscarriages, central nervous system 
disease, and recurrent venous thromboembolism (VTE) in patients with systemic lupus erythematosus (SLE) and serum positivity for anticardiolipin antibodies (aCL) and lupus anticoagulant (LAC). ${ }^{3}$ Recently, Duarte-Garcia et al. found an annual incidence of APS of 2.1 per 100,000 per year, with a prevalence of 50 APS patients per 100,000, equally distributed between males and females. ${ }^{4}$

A more clinically challenging scenario is represented by patients with a clinical history characterized by episodes of thrombosis (especially if recurrent) without cardiovascular risk factors, and more in general, in absence of an identifiable cause of thrombosis, suggestive of a thrombophilic condition, such as APS, but in absence of any positivity of aPL. For these patients, the definition of seronegative APS (SN-APS) was proposed. ${ }^{5}$ In the context of SNAPS, several non-criteria aPL have been investigated with divergent results.

In this review, we will discuss criteria for defining the SN-APS, the new potential non-criteria antibodies implied in SN-APS and its clinical management.

\section{Antiphospholipid syndrome}

\section{Diagnosis of antiphospholipid syndrome}

Antiphospholipid syndrome is a systemic autoimmune disorder characterized by arterial and venous thrombotic manifestations and/or pregnancy morbidity in patients with persistently high levels of aPL. ${ }^{6}$ APS may be classified as primary or secondary, the latter being present in 30$40 \%$ of patients with SLE. The 2006 Sapporo criteria are those currently recommended to diagnose APS. ${ }^{8}$ They include the presence of one clinical criterion and high values of at least one aPL among $\operatorname{IgM} / \mathrm{IgG}$ aCL in serum or plasma, IgM/IgG anti- $\beta 2$ glycoprotein-I (a $\beta 2 \mathrm{GPI})$ antibodies in serum or plasma, and LAC in plasma. Clinical and laboratory criteria are listed in Figure 1 . $^{7}$

The persistence of high antibody values should be tested at least 12 weeks apart, and, in addition, the antibody titers should be dosed at least 12 weeks after the thrombotic event but no more than five years afterwards. ${ }^{7,8}$ Of note, not all patients remain positive over time, and factors associated with persistence of aPL positivity are not well known, but may include inflammation and oxidative stress. ${ }^{9}$

Subjects positive for aPL have a low risk of developing thrombotic events ( $<1 \%$ /year), but after a first episode, the risk of recurrence increases by $10-67 \% .^{10}$ This finding was supported by a recent study performed by Kearon et al., ${ }^{11}$ in which 307 patients with a first unprovoked VTE were tested for aPL. In this study, the persistence of aPL on $\geq 2$ occasions was associated with an increased risk of recurrent thrombosis, despite negative $\mathrm{D}$-Dimer values (HR 4.5: 95\%CI: 1.5-13.0; $P=0.006) .{ }^{11}$

\section{Clinical presentation of antiphospholipid syndrome}

Antiphospholipid syndrome can be broadly classified in venous, arterial or obstetric APS, which are, however, not mutually exclusive. In a retrospective analysis of a cohort of 160 patients with a definite APS, VTE was the most common manifestation (47.5\%), followed by arterial thromboembolism (43.1\%), while obstetrical complications was found in only $9.7 \%$ of patients; in this study, catastrophic APS (C-APS) represented $2.5 \%$ of the cases. ${ }^{12}$

Stroke and transient ischemic attack often involve APS patients, but also lower limb ischemia and myocardial infarction can occur. ${ }^{12}$ In this context, the relationship between aPL and myocardial infarction seems to be bidi-

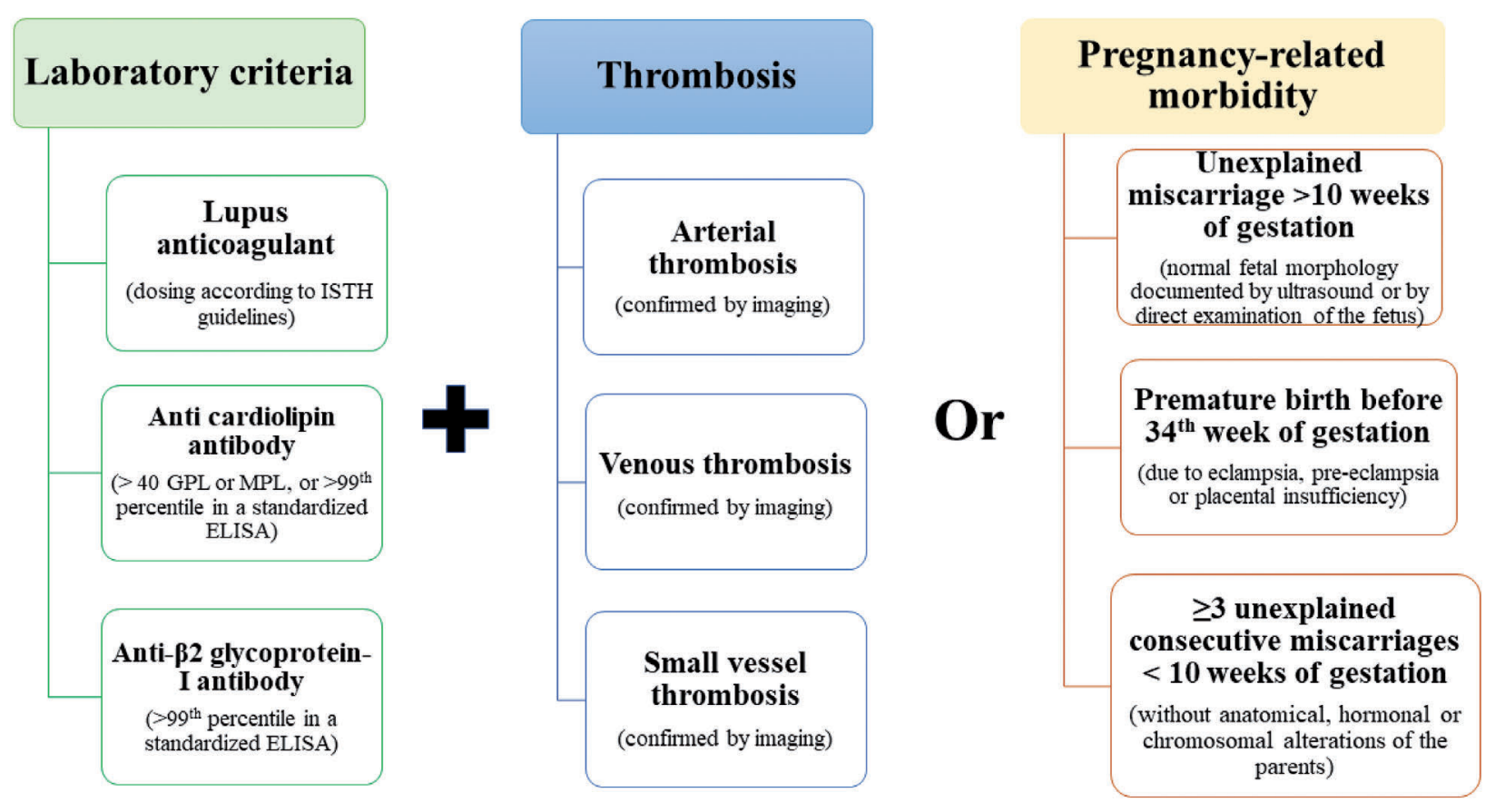

ISTH: International Society on Thrombosis and Haemostasis

Figure 1. Summary of criteria for antiphospholipid syndrome (APS) diagnosis according to Sapporo criteria. GPL: glycopeptidolipid; MPL: monophosphoryl lipid A. 
rectional. Therefore, patients carrying aPL have an increased risk of ischemic heart disease, and conversely, after a first coronary artery disease event, patients positive for aPL showed twice the risk of recurrent major adverse cardiovascular events at 12 and 24 months. ${ }^{13}$ This risk was also evident in subjects with juvenile myocardial infarction in absence of cardiovascular risk factors. ${ }^{13}$

Concerning obstetric complications, fetal loss, especially after $10^{\text {th }}$ week of gestation and premature birth due to eclampsia or placental insufficiency are frequent complications in APS women. ${ }^{14}$ In young women with a history of multiple miscarriage, the immunological study for aPL should be considered.

Finally, catastrophic APS (C-APS) is a severe and lifethreatening manifestation characterized by simultaneous venous or/and arterial thrombosis, often triggered by infections and surgical procedures. C-APS involves multiple organs and systems due to excess of proinflammatory cytokines, coagulation cascade, and platelet activation, leading to thrombosis and microangiopathic hemolytic anemia. ${ }^{15}$

In addition to the above described signs and symptoms, the clinical presentation of patients with APS may be more heterogeneous, involving thrombosis of medium and small vessels (Table 1). ${ }^{6,7,16-18}$ The relevance in clinical practice of non-conventional APS criteria was investigated during the $14^{\text {th }}$ Congress on Antiphospholipid Antibodies, in which each relevant clinical manifestation was analyzed and each evidence was evaluated by the GRADE system. This system also considers the balance of patientimportant outcomes, the overall quality of the evidence for each outcome, and any uncertainty about values. ${ }^{18}$

The most commonly affected sites are the kidney, the skin, and the cardiovascular and nervous systems. In the kidney, it is possible to find an acute thrombotic microangiopathy or a chronic pattern of vaso-occlusive lesions such as cortical ischemic lesions, arterial fibrous intimal hyperplasia or interstitial fibrosis. ${ }^{6}$ APS nephropathy can be identified with a urine test associated with a 24-hour investigation of proteinuria. A biopsy is mandatory in cases where the cause is not clearly identifiable, as in patients with concomitant diabetes, uncontrolled arterial hypertension or other autoimmune diseases such as SLE.

Concerning the skin, livedo reticularis can be found, and recurrent ulcerations called livedoid vasculopathy have also been described. ${ }^{8}$ To evaluate skin abnormalities, a clinical examination is often adequate, and there is usually no need for skin biopsy.

Cardiac abnormalities include valve leaflet thickening, ${ }^{6}$ and diastolic dysfunction, especially of the right ventricle. ${ }^{19}$ Heart valve disease and diastolic dysfunction can be investigated by resting transthoracic echocardiography and by cardiac magnetic resonance imaging (MRI) if a myocardial involvement is suspected (i.e. myocarditis). Pericardium may also be involved, especially in patients with APS and SLE.

Finally, APS is associated with an increased risk of dementia, seizures, multiple sclerosis-like illness, migraine, myelitis transversa and chorea, due to vascular damage and a direct action of antibodies on neurons and ependymal cells., ${ }^{70,21}$ However, unlike other neurological disorders, seizure is not considered a non-conventional criterion due to lack of strength of evidence. ${ }^{18}$ To identify critical illness such as neurological disorders, instrumental examinations are mandatory. An MRI and an electroencephalogram could be useful in recognizing brain atrophy associated with dementia and seizures, and could identify more elusive symptoms such as chorea and migraine if associated with an accurate physical examination.

Other blood alterations include thrombocytopenia (commonly mild with platelet count between $50 \times 10^{9} / \mathrm{L}$ and $150 \times 10^{9} / \mathrm{L}$, but also severe with platelet counts $<20 \times 10^{9} / \mathrm{L}$ often associated with microangiopathy) and hemolytic anemia with the possible presence of schistocytes. ${ }^{6}$ In particular, thrombocytopenia is common in APS, affecting $20-46 \%$ of patients and could paradoxically be associated with an increased risk of thrombosis. ${ }^{18}$ Thrombocytopenia may be the result of an increased activation and destruction of platelets by an immune-mediated mechanism involving aPL or by thrombotic microangiopathy. $^{22}$

After the exclusion of a pseudo-thrombocytopenia, and performing a Coombs test to ascertain the autoimmune nature of thrombocytopenia, corticosteroids, immunosuppressive agents, immunoglobulins and new drugs such as Mammalian target of rapamycin (mTOR) inhibitors and monoclonal antibodies could be helpful in patients with autoimmune thrombocytopenia. ${ }^{23}$

\section{Definition of seronegative antiphospholipid syndrome and non-criteria antiphospholipid antibodies}

The first definition of SN-APS was given in 2003 by Hughes and Khamashta ${ }^{5}$ who described patients with clinical manifestations highly suggestive of APS in absence of the laboratory criteria such as LAC, aCL and aß2GPI antibodies.

Seronegative APS is usually a diagnosis of exclusion and should be suspected in patients with a clinical history suggestive of APS, such as those with recurrent arterial venous thrombotic events, recurrent miscarriage, or unexplained thrombocytopenia, with persistent negativity of aPL tested on at least two occasions, and when other causes of throm-

Table 1. "Extra-criteria" manifestations of antiphospholipid syndrome.

Nervous system

Dementia

Seizures

Multiple sclerosis-like illness

Chorea

Myelitis

\section{Skin}

Livedo reticularis

Livedoid vasculopathy

Heart

Valve vegetations or thickening (Libman-Sacks Endocarditis)

Diastolic dysfunction

Blood

Thrombocytopenia

Hemolytic anemia

Kidney

Microangiopathy

Chronic vaso-occlusive lesions (atherosclerosis, glomerular ischemia, interstitial fibrosis, arterial fibrous intimal hyperplasia) 
bosis are excluded, such as genetic thrombophilia (factor $\mathrm{V}$ and II mutations), active cancer, trauma, major surgery, or prolonged bed rest. This is particularly evident in young patients without established cardiovascular risk factors (i.e. obesity, diabetes, hypertension, dyslipidemia). Most importantly, other forms of coagulopathy should be excluded first, including Protein $\mathrm{C}$ and $\mathrm{S}$ and anti-thrombin deficiency. In addition, the patient's personal medical history should be carefully investigated to exclude previous positivity to aPL.
To better characterize the entity of SN-APS, antibodies against different phospholipids or protein co-factors have been investigated in patients negative to conventional aPL (Table 2).

The antibodies that have been most studied so far are those directed against: 1) a zwitterionic phospholipid, namely phosphatidylethanolamine (PE); 2) negatively charged phospholipids other than cardiolipin, including phosphatidic acid (PA), phosphatidylserine (PS), phosphatidylinositol (PI); 3) vimentin (forming a complex with

Table 2. Summary of positivity for extra-criteria antibodies in each study of seronegative antiphospholipid syndrome (SN-APS).

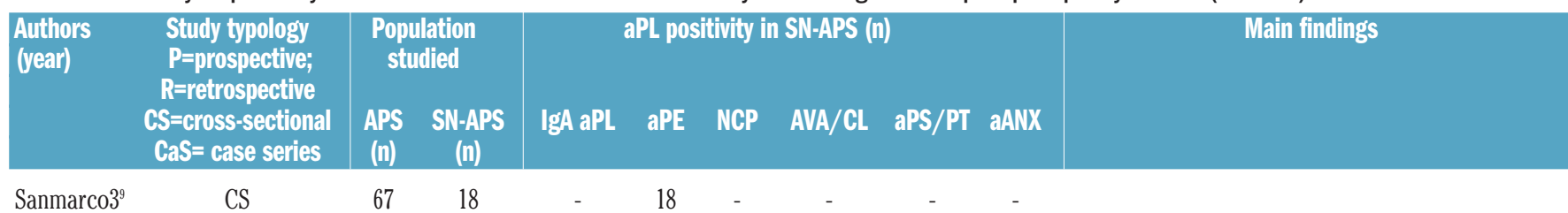

(2001)

\begin{tabular}{|c|c|c|c|c|}
\hline $\begin{array}{l}\text { Sanmarco }{ }^{40} \\
(2007)\end{array}$ & CS & - & 2 & . \\
\hline Kumarrs $^{83}$ & $\mathrm{CaS}$ & - & 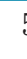 & 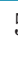 \\
\hline
\end{tabular}

25 of the 40 aPE-positive patients (63\%) were negative for the APS laboratory criteria

Ortona $^{47}$

(2010)

CS $\quad 40 \quad 29 \quad-\quad-\quad-\quad 5$

Conti ${ }^{49}$

(2013)

CS $25 \quad 2$

27 number of mainly SN-APS patients.

SN-APS were positive for 11/24 (45.8\%) for anti-vimentin/cardiolipin antibodies, 3/24 (12.5\%) for anti-prothrombin antibodies, and 1/24 (4.2\%) for anti-annexin $V$ antibodies.

\begin{tabular}{|c|c|c|c|c|c|c|c|c|c|c|}
\hline $\begin{array}{l}\text { Ruiz-García }{ }^{35} \\
(2014)\end{array}$ & CS & 22 & 35 & 35 & - & - & - & - & - & $\begin{array}{l}\text { Isolated IgA a } \beta 2 \mathrm{GPI} \text { antibodies were found in } 22 \% \\
\text { of patients. Patients with arterial thrombosis were } \\
\text { positive only for IgA a } \beta 2 \mathrm{GPI} \text {. }\end{array}$ \\
\hline
\end{tabular}

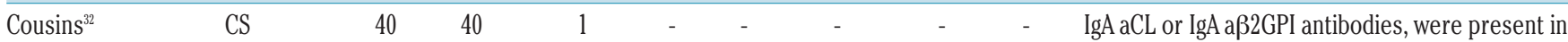

(2015) a significant proportion of patients with APS, and in a small proportion of SN-APS.

\begin{tabular}{llccccccccc}
$\begin{array}{l}\text { Mekiniann }^{27} \\
(2016)\end{array}$ & CS & 83 & 96 & - & 47 & - & & 5 & 57 & $\begin{array}{c}\text { 68\% of patients with obstetrical SN-APS have } \\
\text { non-conventional aPL }\end{array}$ \\
\hline $\begin{array}{l}\text { Zohoury } \\
(2017)\end{array}$ & CS & 107 & 68 & - & 8 & - & 11 & 8 & - & $\begin{array}{c}1 / 3 \text { of SN-APS patients showed reactivity to } 1 \\
\text { or more non-criteria markers }\end{array}$
\end{tabular}

\begin{tabular}{|c|c|c|c|c|c|c|c|c|c|}
\hline $\begin{array}{l}\text { Tortosa }^{36} \\
(2017)\end{array}$ & $\mathrm{R}$ & - & 38 & 38 & - & - & - & - & - \\
\hline
\end{tabular}

The presence of IgA a $\beta 2 \mathrm{GPI}$ in people with no history of APS-events is the main independent risk factor for the development of these types of events, mainly arterial thrombosis

\begin{tabular}{lllllllllll}
\hline $\begin{array}{l}\text { Litvinova }^{25} \\
(2018)\end{array}$ & CS & 41 & 17 & - & - & 5 & - & 4 & 4 & $\begin{array}{r}87 \text { patients: } 41 \mathrm{APS}, 11 \mathrm{aPL} \text { carriers, } 17 \mathrm{SN}-\mathrm{APS} \\
<1 \% \text { of patients with thrombotic/obstetrical }\end{array}$
\end{tabular}
SN-APS had non-conventional aPL

Anti-PS/PT antibodies were correlated with LA. APS triple patients were also positive for anti-PS/PT antibodies

\begin{tabular}{|c|c|c|c|c|c|c|c|c|c|c|}
\hline $\begin{array}{l}\text { Trugliaa } \\
\text { (2018) }\end{array}$ & CS & - & 61 & - & - & - & 33 & - & - & $\begin{array}{l}\text { Non-conventional tests, mainly aCLNim and aCL } \\
\text { seem to be the most sensitive approaches } \\
\text { for identifying aPL in patients with obstetric SN-APS }\end{array}$ \\
\hline $\begin{array}{l}\text { Billoir } \\
(2019)\end{array}$ & $\mathrm{R}$ & - & 23 & - & 23 & - & - & - & - & $\begin{array}{l}\text { aPE persists in } 23 \text { patients (10\%): } 15 \text { with } \\
\text { a thrombotic event, } 6 \text { with obstetrical morbidity } \\
\text { and } 2 \text { with a combined event }\end{array}$ \\
\hline $\begin{array}{l}\text { Ganapati }^{61} \\
\text { (2019) }\end{array}$ & $\mathrm{R}$ & 58 & 12 & - & - & - & - & 7 & - & $\begin{array}{c}\text { Addition of aPS/PT to current APS criteria to SN-APS } \\
\text { patients led to reclassification of additional } 12.1 \% \\
\text { patients as APS overall and } 42.8 \% \text { in obstetric } \\
\text { APS category }\end{array}$ \\
\hline
\end{tabular}

aANX: Annexin A5 antibody; aPE: phosphatidylethanolamine; aPL: antiphospholipid antibodies; APS: antiphospholipid syndrome; aPS/PT antiphosphatidylserine/prothrombin; AVA/CL: anti vimentin/cardiolipin complex; LA: lupus anticoagulant; NCP: negatively charged phospholipids (phosphatidic acid, phosphatidylserine and phosphatidylinositol); SN-APS: seronegative APS 
cardiolipin); 4) prothrombin (forming complex with PS anti-PS/PT); and 5) the anticoagulant protein Annexin A5 (Table 2). In addition, the IgA isotype a $\beta 2 \mathrm{GPI}$ is under investigation in APS and SN-APS patients.

A collaborative USA/UK study analyzed a comprehensive panel of 'non-criteria' aPL tests in a series of 175 consecutive patients matching the criteria for APS and $68 \mathrm{SN}$ APS patients with clinical manifestations suggestive of APS but having negative serology. The Authors found that one-third of the 'seronegative' sera gave positive results. ${ }^{24}$ The study concluded that patients with clinical features of APS, but negative for conventional criteria markers, should undergo additional testing for non-criteria biomarkers. ${ }^{24}$

A recent study evidenced that positivity for the extracriteria aPL was $<1 \%$ in SN-APS (thrombotic or obstetric); however, the lack of clear inclusion and exclusion criteria does not allow a precise estimation of the prevalence to be made..$^{25}$

Similarly, non-criteria antibodies were detected in $18.8 \%$ of SN-APS patients also in a Chinese cohort composed of APS and patients with only clinical criteria for APS. ${ }^{26}$ This is also confirmed in obstetric SN-APS patients, in whom $68 \%$ were positive for non-conventional aPL. ${ }^{27}$

Here we will provide an overview of current evidence on the most studied non-criteria aPL that, although not validated in large cohort studies, may have a potential role in the pathogenesis of APS.

\section{IgA antibody isotype anti- $\beta 2$ glycoprotein-I and anticardiolipin antibodies}

There is a growing body of evidence to suggest a potential usefulness of IgA in the context of APS. ${ }^{28}$ Very recent evidence suggested that, while IgG/M isotypes recognize an epitope in domain 1, the epitopes recognized by IgA are the domains 3,4 and $5 .{ }^{29}$ However, as reported by the $13^{\text {th }}$ International Congress on Antiphospholipid Antibodies, testing for IgA-a $\beta 2$ GPI should be considered only in patients negative for IgG and IgM isotypes with APS symptoms. ${ }^{30}$

Studies investigating the prevalence of IgA aPL reported a variable prevalence ranging from $14 \%$ to $72 \%$ according to different reports ${ }^{28}$ however, these studies have a retrospective design, used different assays to measure IgA aPL, and used different cut-off values to define aPL positivity. ${ }^{31,32}$

A large study including 5,892 patients (803 with SLE and 5,089 from the Antiphospholipid Standardization Laboratory sent for evaluation for APS) found that IgA aß2GPI isotype was positive in 255 (4.3\%) patients, in 198 cases in association with other aPL, while only aPL was detectable in $57 \cdot{ }^{33}$ Isolated IgA a $\beta 2$ GPI positivity was associated with an increased risk of arterial thrombosis $(P<0.001)$, venous thrombosis $(P=0.015)$, and all thrombosis $(P<0.001) .^{33}$

A second study evaluated, in addition to $\operatorname{IgM}$ and $\operatorname{IgG}$, the positivity and predictivity of IgA aCL and IgA a $\beta 2 \mathrm{GPI}$ in 430 patients: 111 with APS, 119 with SLE, and 200 healthy controls. ${ }^{34}$ Positivity for IgA aCL was $38 \%$; IgA a $\beta 2 \mathrm{GPI}$ was $46 \%$ in patients with APS. All three antibody isotypes (IgM, IgG and $\operatorname{IgA}$ ) were significantly associated with a diagnosis of APS, with high specificity but not good sensitivity, based on receiver operating characteristic (ROC) analysis. Looking at likely hazard ratios, the IgA aß2GPI (HR 33.9, 95\%CI: 10.5-109.5) was similarly asso- ciated to APS as compared to IgG a $32 \mathrm{GPI}$ (HR 33.4, 95\% CI: 13.0-86.1), but showed a higher association compared to IgM aß2GPI (HR 9.2, 95\%CI: 4.6-18.4) and was associated with thrombotic but not obstetric complications in patients with APS. ${ }^{34}$

Indeed, IgA a $32 \mathrm{GPI}$ levels seem to be associated with thrombotic events in patients without other aPL. . $^{33,35}$ Thus, a case-control study including 244 asymptomatic patients screened for aPL and positive only for IgA a 2 GPI and 221 negative patients followed for five years showed that the presence of IgA a $32 \mathrm{GPI}$ was associated with an increased risk for developing clinical thrombotic APS events (OR 5.15; $P<0.001)^{36}$

Although attractive, these data were not confirmed by another study evaluating the presence of IgA a $\beta 2$ GPI antibodies in SN-APS. ${ }^{25}$

Based on this evidence, it is not clear whether testing for $\operatorname{IgA~aCL}$ and IgA a 2 GPI antibodies in addition to the routine tests may improve thrombotic risk stratification. Thus, the use of IgA antibodies to identify a SN-APS needs to be further investigated.

\section{Antibodies to phosphatidylethanolamine}

Phosphatidylethanolamine is mainly found in the inner leaflets of plasma membranes and contributes to $20-50 \%$ of total phospholipids. It works as an anticoagulant by enhancing activated protein C (APC) activity. Other investigators have demonstrated that $\mathrm{PE}$ inhibits coagulation activity interfering with the factor Xa-prothrombin system..$^{37}$

Several studies reported that antibodies against PE (aPE) are significantly associated with major clinical events such as fetal loss and/or thrombosis, and are mainly present in the absence of the laboratory criteria of APS. Bérard et al. showed that aPE were the only aPL found in 6 of 34 patients suffering from thrombotic events and with a negative screening for antibodies to anionic phospholipids, including LA ${ }^{38} \mathrm{~A}$ second study focused on patients with unexplained thrombosis and no criteria for APS. Thus, in 98 patients with unexplained thrombosis, 142 with thrombophilia, 67 with APS, 75 with hereditary hemostatic defects and 110 without thrombosis, the authors found that aPE prevalence was significantly higher both in patients with APS $(43 \% ; P<0.0001)$ and in those with unexplained thrombosis $(18 \% ; P=0.001)$ compared to patients without thrombosis. ${ }^{39}$ Subsequently, in a large multicenter study including 317 patients with deep venous thrombosis and 52 with arterial events, aPE were found in $15 \%$ of the thrombotic patients, most of whom were only positive for $\mathrm{aPL} .{ }^{40}$ Some interesting data were also reported regarding the association between aPE and obstetric complications. Gris et al. measured various aPL in a large cohort of 518 women with unexplained or explained early fetal losses and a control group of healthy mothers. IgM-aPE were found to be independent risk factors for unexplained early fetal loss. ${ }^{41}$

A retrospective study on 228 SN-APS demonstrated a positivity for aPE in $10 \%$ of the patients. ${ }^{42}$ In contrast, a recent study on a Chinese population composed of APS and patients with only clinical criteria for APS failed to demonstrate any aPE positivity in SN-APS. ${ }^{26}$

The above reported results suggest that aPE could be considered as markers of a variant of APS when they are associated with thrombosis and a potential tool to define the SN-APS subjects. 
Antibodies against phosphatidic acid, phosphatidylserine and phosphatidylinositol

In an effort to expand the panel of aPL to other negatively-charged phospholipids, antibodies against phosphatidic acid (PA), phosphatidylserine (PS), and phosphatidylinositol (PI), which fall under the category of anionic phospholipids, were proposed. ${ }^{43}$ Anti-PS antibodies inhibited the development and invasion of the trophoblast, decreased hCG levels, and retarded the formation of syncytiotrophoblast in in vitro models. ${ }^{44} \mathrm{Few}$ clinical studies have investigated this issue. In a first study on 866 women with recurrent pregnancy loss (RPL), the authors found that 87 of 866 women who were negative for aCL had a positivity for one of the other aPL. ${ }^{42}$ In a second study on 872 women with RPL, 49 (3.6\%) were negative for both aCL and LA but positive for aPS. ${ }^{46}$ In this second study, the presence of aPS had a positive correlation with the number of consecutive pregnancy losses. ${ }^{46}$ This result was not confirmed when the same author analyzed a larger population of 1,020 woman with RPL. ${ }^{46}$ Moreover, Zhang et al. did not find any positivity for aPE, aPS or aPI in an evaluation study of 288 subjects (86 patients with APS, 30 patients with non-APS thrombosis, 32 patients with non-APS pregnancy-related morbidity, 42 patients with SLE, and 39 healthy controls). ${ }^{26}$

Based on the current evidence, testing for aPA, aPI and aPS is not recommended, as these antibodies appeared to overlap with the accepted diagnostic markers of APS. Nonetheless, the results obtained on RPL with a seronegative profile suggest a potential role for aPA, aPI and aPS in defining SN-APS in this particular setting.

\section{Anti-vimentin/cardiolipin complex}

Vimentin is the most abundant type III intermediate filament of the cytoskeletal system and it was recently localized on the surface of apoptotic neutrophils and T cells, activated macrophages, platelets, and vascular endothelial cells. After becoming antigenic with a still unexplained mechanism, Vimentin is exposed and could be bound by anti-vimentin antibodies (AVA). ${ }^{47,48}$ Vimentin could also electrically interact with cardiolipin on the surface of apoptotic cells generating the vimentin/cardiolipin complex. Antibodies against this complex (vimentin/cardiolipin antibodies, AVA/CL) show a prothrombotic effect. Thus, Ortona et al. demonstrated an AVA/CL-mediated activation of the TLR4/IRAK/Nf-kB molecular pathway that leads to the release of pro-inflammatory and procoagulant factors by endothelial cells. ${ }^{47}$ Hence, AVA/CL could play a role in arterial thrombosis by inducing platelet and coagulation cascade activation.

The role of AVA/CL in SN-APS has been investigated only in a few clinical studies. Thus, Ortona et al..$^{47}$ analyzed serum IgG AVA/CL antibodies detected by ELISA in 29 SN-APS, 40 APS, 30 patients with SLE, 30 with rheumatoid arthritis, and 32 healthy controls. They found a positivity for AVA/CL in almost all APS patients $(92 \%)$, and also in a large proportion of SN-APS (55\%); interestingly, this positivity was persistent in almost all cases. Similarly, Conti et al. found AVA/CL positivity in $24 \mathrm{SN}$ APS patients. ${ }^{49}$ Moreover, in a retrospective analysis of 61 obstetric SN-APS, $76 \%$ resulted positive for AVA/CL. ${ }^{50}$ However, the overlapping presence of AVA/CL antibodies in SLE and APS weakens the specificity of such a diagnos- tic marker. Hence, the observation by Ortona et al. ${ }^{47}$ needs to be confirmed by larger prospective clinical studies in order to better define the role of AVA/CL in SN-APS.

\section{Anti-prothrombin and antiphosphatidylserine/prothrombin antibodies}

Prothrombin is a plasma glycoprotein involved in the coagulation cascade converted to thrombin by extrinsic thromboplastin during the second stage of blood clotting. ${ }^{51}$ A large amount of data, obtained from various, mainly retrospective, studies gave contrasting evidence concerning the clinical significance of anti-prothrombin antibody (aPT). Thus, in a comparison between 106 subjects who experienced either a non-fatal myocardial infarction or cardiac death and 106 subjects without coronary disease, Vaarala et al. found that a high level of aPT (highest tertile of distribution) predicted a 2.5 -fold increase in the risk of cardiovascular events. ${ }^{52}$ Conversely, Atsumi et al. did not find any correlation between clinical manifestation of aPT and APS in an evaluation of 265 APS patients. ${ }^{53}$ More recently, two prospective studies validated the role of aPT in predicting the first or recurrent risk of thrombosis in patients with APS..$^{54,55}$ Considering a group of 142 LA positive patients, Forastiero et al. found that a higher rate of thrombosis in patients with positive anti-PT compared with patients without anti-PT (8.6\% vs. 3.5\% per patient year). The highest incidence of thrombosis was detected in patients positive for both a $32 \mathrm{GPI}$ and $\mathrm{aPT}(8.4 \%$ per patient year). ${ }^{54}$ Moreover, a 15-year longitudinal prospective study by Bizzaro et al. identified IgG aPT antibody as the most useful thrombosis predictor in SLE patients. ${ }^{55}$

Another intriguing issue is represented by the different potential role of IgG/IgM antiphosphatidylserine/prothrombin (aPS/PT) compared to aPT. Indeed, a high correlation between APS classical antibody panel and aPS/PT IgG/IgM suggests that this marker may be useful in the evaluation of APS. ${ }^{56}$ The clinical significance of aPT and aPS-PT was evaluated by testing for the presence of these antibodies in 212 SLE patients and in 100 healthy individuals. Results show that aPT and aPS-PT were found in $47 \%$ of the patients (aPT in $31 \%$ and aPS-PT in 31\%). Their presence did not correlate with that of aCL, a $32 \mathrm{GPI}$, LA and/or anti-protein S. IgG but not IgM aPT were more frequently found in patients with thrombosis than in those without. IgG and IgM aPS-PT were also more frequent in patients with thrombosis (venous and/or arterial) than in those without. Levels of IgG aPT and IgG and IgM aPS-PT were higher in patients with thrombosis than in those without. More significantly, $48 \%$ of the patients with aPL-related clinical features who were negative for standard tests had aPT. ${ }^{77}$ Recently, the clinical significance of aPS/PT antibodies was prospectively evaluated in a cohort of 191 aPL carriers: ${ }^{58} \mathrm{IgG}$ aPS/PT antibodies were detected in $40(20.9 \%)$ and IgM aPS/PT in $102(53.4 \%)$ of the carriers. The cumulative incidence rate of thrombotic events was significantly higher in the IgG aPS/PT positive $(P=0.035)$ but not in the IgM aPS/PT positive carriers. Similar results were obtained in a second study evaluating 152 patients with a previous thrombosis of whom 90 were SN-APS; $10 \%$ of SN-APS patients in this study were positive for aPS/PT.$^{59}$ Of note, aPS/PT are associated with recurrent early or late abortions and with premature delivery irrespective of other aPL. ${ }^{60}$

Based on the above studies, aPT and aPS/PT can be 
potentially used as confirmatory diagnostic markers and as indicators of the risk of thrombosis. Recently, the presence of $\operatorname{IgG}$ and IgM aPS/PT was also detected in 9 of 17 $\mathrm{SN}-\mathrm{APS}{ }^{25}$ Similar, and even stronger evidence was provided by two retrospective studies on SN-APS patients that found approximately $50 \%$ of subjects were positive for aPS/PT. ${ }^{61,62}$ Nonetheless, further studies must be undertaken before these antibodies can be included in the diagnostic criteria of SN-APS.

\section{Annexin A5 antibody}

Annexin A5 is a glycoprotein that binds to negative phospholipids such as PS. It has been proposed that annexin A5 forms a protective anticoagulant shield on vascular endothelial cells and that a $32 \mathrm{GPI}$ antibodies in complex with B2GPI may disturb the shield and hence predispose to thrombosis. Due to this marked heterogeneity, it remains controversial whether anti-annexin A5 antibodies (aANX) are associated with clinical manifestations. In a comparison of 112 APS patients with 40 healthy controls, Singh et al. found aANX positivity in 69 APS and in only 3 controls. ${ }^{63}$ On the contrary, de Laat et al. found no association between aANX and history of thrombosis in 198 patients with primary APS, SLE or lupus-like disease. ${ }^{64}$ aANX was also found to be predictive for fetal loss in a study of three groups (total 518 women) of patients with unexplained primary recurrent early fetal loss, patients with explained episodes, and mothers with no previous obstetric incident, respectively. ${ }^{41}$

Annexin A5 resistance was proposed as a mechanism for APS. The annexin A5 resistance (A5R) assay identifies patients with an antibody-mediated disruption of annexin A5 on endothelial surfaces. This is demonstrated by the resistance to the annexin A5 anticoagulant effects (i.e. annexin A5 resistance) in vitro. This test was validated in 750 patients with a history of thrombosis, pregnancy complications, and controls. ${ }^{65}$ The authors found a reduction in A5 anticoagulant ratios in aPL antibody-positive patients with thrombosis and/or pregnancy complications compared with aPL antibody-negative patients and controls. This suggests that reduced A5R could identify patients with a propensity for thrombosis or pregnancy complications. $^{65}$

Very recently, a case report described the presence of multiple annexin autoantibodies in a patient with recurrent miscarriages, fulminant stroke, and SN-APS. ${ }^{6}$ Hence, although attractive, the evaluation of aANX or A5R in clinical practice for the management of SN-APS requires larger prospective studies.

\section{From risk assessment to antithrombotic treatment}

The first step in the assessment of thrombotic risk in APS and SN-APS patients is represented by antibody characterization and evaluation of cardiovascular risk factors. Thus, the thrombotic risk varies according to aPL positivity and antibody titers. For example, a retrospective study on 3,088 APS patients demonstrated that single positivity for aCL or a $\beta 2 \mathrm{GPI}$ was associated with low risk of event [odds ratio $(\mathrm{OR})<5$ ], while LA positivity alone conferred a medium risk of event (OR 5-9); this risk increased in patients with double or triple positivity $(\mathrm{OR}>9){ }^{67}$

Some scores have also been proposed to stratify the risk of events in APS patients; the APL Score has no clinical items and is based exclusively on the antibody titers (Table 3). ${ }^{68}$ An aPL score of $\geq 30$ was an independent risk factor for thrombosis (hazard ratio 3.144, 95\% CI: 1.383 7.150; $P=0.006$ ) in patients with autoimmune diseases. ${ }^{69}$

Another score is the Global Anti-Phospholipid Syndrome Score (GAPSS), which was developed in a cross-sectional study on a cohort of 211 patients with SLE. The score includes traditional cardiovascular risk factors such as hypertension and hyperlipidemia and the presence / absence of aPL70 (Table 3). Of note, both scores also include one non-criteria aPL, such as aPS/PT.

These scores, although potentially useful in clinical practice, require further prognostic validation.

The thrombotic risk stratification is more challenging in patients with SN-APS. It is important to identify and characterize the presence of non-criteria $\mathrm{aPL}$, as they seem to be associated with different thrombotic complications (Table 4). Thus, aPS/PT and antibodies to vimentin/CL complex increase the risk of arterial thrombosis, while pregnancy-related complications are associated with the presence of PE, PA, PS and PI antibodies (Table 4).

Table 3. Scores for risk stratification in antiphospholipid syndrome.

\begin{tabular}{lcc} 
APL Score - IIEMS & CUT-0FF & POINIS \\
APTT mixing $>49$ sec & 5 & \\
CONFIRMATION TEST, ratio & $>1.3$ & 2 \\
& $>1.1$ & 1 \\
\hline KCT mixing $>29 \mathrm{sec}$. & 8 & \\
DRWT mixing & $>45 \mathrm{sec}$. & 4 \\
\hline CONFIRMATION TEST, ratio & $>1.3$ & 2 \\
& $>1.1$ & 1 \\
IgG ACL, GPL. & $>30$ & 20 \\
High titers & $>18.5$ & 4 \\
Medium/low titers & $>7$ & 2 \\
\hline IgM ACL, MPL & 20 & \\
IgG Anti- $\beta 2 \mathrm{GPI}$ & $>2.2 \mathrm{U}$ & 6 \\
High titers $>15 \mathrm{U}$ & $>6 \mathrm{U}$ & 1 \\
Medium/low titers & & \\
\hline IgM anti- $\beta 2 \mathrm{GPI}$ & 20 & \\
IgG aPS/PT & $>2 \mathrm{U}$ & 13 \\
High titers $>10 \mathrm{U}$ & 8 & \\
Medium/low titers &
\end{tabular}

IgM aPS/PT $>9.2 \mathrm{U}$

\begin{tabular}{lcc} 
GAPSS & Item & Points \\
CLINICAL & Hyperlipidemia & 3 \\
& Arterial hypertension & 1 \\
LABORATORY & aCL IgG/IgM & 5 \\
& Anti $\beta 2 \mathrm{GPI}$ IgG/IgM & 4 \\
aPS/PT IgG/IgM & 3 \\
LA & 4 \\
Total & 20 \\
\hline
\end{tabular}

APTT: activated partial thromboplastin time; KCT: kaolin clotting time; dRVVT: dilute Russell's viper venom time; aCL: anticardiolipin antibodies; $\beta 2 \mathrm{GPI}$ : $\beta 2$-glicoprotein I; aPS/PT: phosphatidylserine prothrombin complex; aPL: antiphospholipid antibody GPL: IgG phospholipid units; MPL: IgM phospholipid units; LA: lupus anticoagulant. 


\section{Primary and secondary prevention strategies}

\section{Antiphospholipid antibody carriers}

In subjects with positivity for aPL in the absence of clinical thrombotic events, primary prevention strategy includes cardiovascular risk factors such as arterial hypertension, diabetes, dyslipidemia and cigarette smoking (Figure 2). Treatment with low-dose aspirin (LDA, 75-100 $\mathrm{mg} / \mathrm{die}$ ) is still controversial ${ }^{6}$ and could be considered in patients at high risk, such as those with triple positivity or persistent positivity with medium-high titer of $\mathrm{aCL} .^{71,72}$ Recently, the positivity for IgG aPS/PT has been suggested as a marker of thrombotic risk in aPL carriers in addition to triple positivity (Figure 2) ${ }^{58}$ Regarding oral anticoagulation, with or without LDA, the quality of evidence is too low to demonstrate benefit or harm of anticoagulant use in aPL carriers. ${ }^{73}$
In women with a high-risk aPL profile but no history of thrombosis or pregnancy complications, treatment with LDA (75-100 mg daily) during pregnancy should be considered according to 2019 European League Against Rheumatism (EULAR) recommendations.

\section{Secondary antiphospholipid syndrome}

In APS patients with previous arterial or venous thromboembolism, use of unfractionated or low molecular weight heparins (LMWH) is recommended in the acute phase $^{6}$ followed by long-term treatment with warfarin, with an international normalized ratio (INR) range between 2-3.8.74

Warfarin therapy in APS has several critical points. Indeed, a recent study ${ }^{10}$ showed that, in the APS population, the management of anticoagulant therapy is more problematic compared to a population of patients with

Table 4. Suggested extra-criteria antibodies in seronegative antiphospholipid syndrome and its clinical manifestations.

Extra-critera anthodiles Clinical manifiestations

Anti-prothrombin/phosphatidylserine antibodies

Anti-annexin $\mathrm{V}$ antibodies/annexin $\mathrm{A} 5$ resistance

Antibodies to vimentin/CL complex

Phosphatidylethanolamine

Phosphatidic acid

Phosphatidylserine

Phosphatidylinositol

IgA aCL and $\mathrm{a} \beta 2 \mathrm{GPI}$ antibodies

CL:cardiolipin; a $\beta 2$ GPI: anti- $\beta 2$ Glycoprotein I.
Thrombosis

Thrombosis and/or pregnancy complications

Arterial thrombosis

Fetal loss and/or thrombosis

Fetal loss

Fetal loss

Fetal loss

Thrombosis

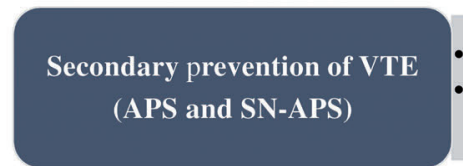

\section{LMWH or UFH}

Warfarin (INR range 2.0-3.0), If recurrence under warfarin, add on statin, LDA or hydroxychloroquine

Secondary prevention in arterial thrombosis

(APS and SN-APS)

\section{LDA/Clopidogrel}

Warfarin (INR range 2.0-3.0). Consider LDA association in patients with recurrent arterial thrombosis and low risk of bleeding.

- Add statin if indicated

Figure 2. Summary of antithrombotic treatment options in patients with antiphospholipid syndrome and seronegative antiphospholipid syndrome. ${ }^{72}$ APS: antiphospholipid syndrome; aPL: antiphospholipid antibodies; aCL: anticardiolipin antibodies; VTE: venous thromboembolism; LDA: Iow-dose aspirin, LMWH: Iow molecular weight heparin, UFH: unfractionated heparin; INR: international normalized ratio; EULAR: European League Against Rheumatism. 
atrial fibrillation (AF). Thus, APS patients had a shorter time within the therapeutic range than those with $\mathrm{AF}$ (53.5\% vs. 68\%; $P=0.001)$ and needed a higher mean weekly dose of warfarin to reach the therapeutic range. ${ }^{10}$

In the case of low-quality therapy with warfarin or recurrent thrombosis, two possible therapeutic approaches could be considered. The first is to adopt a higher intensity warfarin therapy with target INR 3-4, which is, however, not current practice given its association with a reduced risk of thrombosis in the majority of patients. ${ }^{6,7275} \mathrm{~A}$ second approach is represented by the addition of LDA to anticoagulation, which should, however, be reserved for highrisk patients, particularly after an arterial thrombotic event. ${ }^{6,76}$

More recently, non-vitamin $\mathrm{K}$ antagonist oral anticoagulants (NOAC) have been investigated in patients with APS with divergent results. ${ }^{77}$ Following the results from the Trial on Rivaroxaban in AntiPhospholipid Syndrome (TRAPS), ${ }^{78}$ which included triple positive thrombotic APS, rivaroxaban is contraindicated in APS patients with triple aPL positivity. $^{72}$ An analysis from the RE-COVER/RE-COVER II and RE-MEDY trials showed similar safety and efficacy of dabigatran in patients with thrombophilia and previous venous thromboembolic events, in whom APS represented the second most common inherited disorders, accounting for $20 \%$ of all patients. ${ }^{79}$ These results need to be confirmed in realworld studies. A randomized trial investigating the efficacy and safety of apixaban in APS patients is currently ongoing $;^{80}$ this study will include patients with both venous and arterial thrombosis. Laboratory testing of NOAC may be useful in patients with APS as no pre-clinical data in this patient population are available.

Recently, new drugs have been administered in APS patients with thrombotic events. A first example is represented by mTOR inhibitors; these were found to reduce the onset of new vascular lesions after transplantation in patients with APS nephropathy. ${ }^{81}$ Monoclonal antibodies such as rituximab ${ }^{22}$ (anti-CD20 agent) and eculizumab ${ }^{23}$ (anti-C5 agent) are currently administrated to manage noncriteria symptoms refractory to standard therapy and to add-on in catastrophic APS and kidney transplantation in APS patients, respectively. Despite these findings, the use of these drugs should be avoided due to lack of strong evidence in APS patients; their use could be considered in patients with refractory C-APS, as suggested by EULAR guidelines. ${ }^{72}$

\section{Obstetric antiphospholipid syndrome}

Women who are diagnosed with confirmed APS should be treated with antepartum administration of prophylactic or intermediate dose of unfractionated heparin or prophylactic LMWH combined with LDA (75-100 mg/day), according to the 2012 American College of Cardiology (ACC) guidelines. ${ }^{74}$

Recent 2019 EULAR recommendations suggest that women: 1) with a history of obstetric APS, such as a history of $\geq 3$ recurrent spontaneous miscarriages $<10^{\text {th }}$ week of gestation and in those with a history of fetal loss $\left(\geq 10^{\text {th }}\right.$ week of gestation); and 2) with a history of delivery $<34$ weeks of gestation due to eclampsia/severe pre-eclampsia or due to placental insufficiency, should be started on a combined therapy including LDA and prophylactic heparin during pregnancy. ${ }^{72}$

Heparin at prophylactic dose should be maintained for six weeks after delivery to avoid maternal thrombosis. Finally, heparin should be increased to therapeutic doses, in addition to LDA, in women with a history of thrombotic APS.

\section{Conclusions}

The diagnosis of SN-APS should be formulated only after the exclusion of other causes of inherited and acquired thrombophilic conditions. Although several different antibodies to a number of antigens are involved in $\mathrm{SN}$-APS, the routine testing of these non-criteria antibodies is not recommended, but may be considered in patients with a high clinical suspicion of APS, such as those presenting with recurrent unexplained thrombosis, thrombosis at unusual sites, or women with recurrent pregnancy-related complications. The assessment and interpretation of these non-conventional antibodies should be performed by specialized centers of hemostasis and thrombosis to reduce laboratory variability.

The detection of non-criteria aPL may help guide antithrombotic strategies in SN-APS patients with arterial or venous thrombosis. As an example, patients treated with NOAC for recurrent VTE events, who become positive for non-criteria aPL, may be switched to VKA or LMWH, especially in cases of a recurrent thrombotic events. Moreover, in case of an unprovoked DVT, and among patients who could be withdrawn from anticoagulation, the positivity for a non-criteria aPL may help decide whether or not to continue long-term anticoagulation.

In conclusion, there is growing evidence to suggest a role for non-criteria aPL in those patients defined as "seronegative".

\section{References}

1. Meroni PL, Borghi MO, Raschi E, Tedesco F. Pathogenesis of antiphospholipid syndrome: understanding the antibodies. Nat Rev Rheumatol. 2011;7(6):330-339.

2. Andreoli L, Chighizola CB, Banzato A, et al. Estimated frequency of antiphospholipid antibodies in patients with pregnancy morbidity, stroke, myocardial infarction, and deep vein thrombosis: a critical review of the literature. Arthritis Care Res (Hoboken). 2013;65(11):1869-1873

3. Hughes GR. Thrombosis, abortion, cerebral disease, and the lupus anticoagulant. Br Med J (Clin Res Ed). 1983;287(6399):1088-1089.

4. Duarte-Garcia A, Pham MM, Crowson CS, et al. The Epidemiology of Antiphospholipid Syndrome. A PopulationBased Study. Arthritis Rheumatol. 2019;71 (9):1545-1552.

5. Hughes GR, Khamashta MA. Seronegative antiphospholipid syndrome. Ann Rheum Dis. 2003;62(12):1127.

6. Garcia D, Erkan D. Diagnosis and Management of the Antiphospholipid Syndrome. N Engl J Med. 2018;378(21): 2010-2021.

7. Limper M, de Leeuw K, Lely AT, et al.
Diagnosing and treating antiphospholipid syndrome: a consensus paper. Neth J Med. 2019;77(3):98-108.

8. Miyakis S, Lockshin MD, Atsumi $\mathrm{T}$, et al. International consensus statement on an update of the classification criteria for definite antiphospholipid syndrome (APS). J Thromb Haemost. 2006;4(2):295-306.

9. Lopez-Pedrera C, Barbarroja N, JimenezGomez Y, et al. Oxidative stress in the pathogenesis of atherothrombosis associated with anti-phospholipid syndrome and systemic lupus erythematosus: new therapeutic approaches. Rheumatology (Oxford). 2016;55(12):2096-2108. 
Seronegative antiphospholipid syndrome

10. Pastori D, Parrotto S, Vicario T, et al. Antiphospholipid syndrome and anticoagulation quality: a clinical challenge. Atherosclerosis. 2016;244:48-50.

11. Kearon C, Parpia S, Spencer FA, et al. Antiphospholipid antibodies and recurrent thrombosis after a first unprovoked venous thromboembolism. Blood. 2018;131(19): 2151-2160.

12. Pengo V, Ruffatti A, Legnani C, et al. Clinical course of high-risk patients diagnosed with antiphospholipid syndrome. J Thromb Haemost. 2010;8(2):237-242.

13. Pastori D, Bucci T, Triggiani $M$, et al. Immunoglobulin $\mathrm{G}$ ( $\operatorname{Ig} G$ ) anticardiolipin antibodies and recurrent cardiovascular events. A systematic review and Bayesian meta-regression analysis. Autoimmun Rev. 2019;18(5):519-525

14. Uthman I, Noureldine MHA, Ruiz-Irastorza G, Khamashta M. Management of antiphospholipid syndrome. Ann Rheum Dis. 2019;78(2):155-161.

15. Espinosa G, Rodriguez-Pinto I, Cervera R. Catastrophic antiphospholipid syndrome: an update. Panminerva Med. 2017;59(3): 254-268.

16. Espinosa G, Cervera R. Current treatment of antiphospholipid syndrome: lights and shadows. Nat Rev Rheumatol. 2015;11(10): 586-596.

17. Schreiber K, Radin M, Sciascia S. Current insights in obstetric antiphospholipid syndrome. Curr Opin Obstet Gynecol. 2017;29(6):397-403.

18. Abreu MM, Danowski A, Wahl DG, et al. The relevance of "non-criteria" clinical manifestations of antiphospholipid syndrome: 14th International Congress on Antiphospholipid Antibodies Technical Task Force Report on Antiphospholipid Syndrome Clinical Features. Autoimmun Rev. 2015;14(5):401-414.

19. Tektonidou MG, Ioannidis JP, Moyssakis I, et al. Right ventricular diastolic dysfunction in patients with anticardiolipin antibodies and antiphospholipid syndrome. Ann Rheum Dis. 2001;60(1):43-48

20. Roldan JF, Brey RL. Neurologic manifestations of the antiphospholipid syndrome. Curr Rheumatol Rep. 2007;9(2):109-115.

21. Bucci T, Menichelli D, Pignatelli P, et al. Relationship of Antiphospholipid Antibodies to Risk of Dementia: A Systematic Review. J Alzheimers Dis. 2019;69(2):561-576.

22. Artim-Esen B, Diz-Kucukkaya R, Inanc $M$. The significance and management of thrombocytopenia in antiphospholipid syndrome. Curr Rheumatol Rep. 2015;17(3):14

23. Chighizola CB, Meroni PL. Thrombosis and Anti-phospholipid Syndrome: a 5-Year Update on Treatment. Curr Rheumatol Rep. 2018;20(7):44.

24. Zohoury N, Bertolaccini ML, RodriguezGarcia JL, et al. Closing the Serological Gap in the Antiphospholipid Syndrome: The Value of "Non-criteria" Antiphospholipid Antibodies. J Rheumatol. 2017;44(11):15971602.

25. Litvinova E, Darnige L, Kirilovsky A, et al. Prevalence and Significance of Non-conventional Antiphospholipid Antibodies in Patients With Clinical APS Criteria. Front Immunol. 2018;9:2971.

26. Zhang S, Wu Z, Zhang W, et al. Clinical performance of non-criteria antibodies to phospholipids in Chinese patients with antiphospholipid syndrome. Clin Chim Acta. 2019;495:205-209.

27. Mekinian A, Bourrienne MC, Carbillon L, et al. Non-conventional antiphospholipid antibodies in patients with clinical obstetrical APS: Prevalence and treatment efficacy in pregnancies. Semin Arthritis Rheum. 2016;46(2):232-237.

28. Brusch A. The Significance of Anti-Beta-2Glycoprotein I Antibodies in Antiphospholipi
(Basel). 2016;5(2)

29. Serrano M, Martinez-Flores JA, Norman GL, et al. The IgA Isotype of Anti-beta2 Glycoprotein I Antibodies Recognizes Epitopes in Domains 3, 4, and 5 That Are Located in a Lateral Zone of the Molecule (LShaped). Front Immunol. 2019;10:1031.

30. Lakos G, Favaloro EJ, Harris EN, et al. International consensus guidelines on anticardiolipin and anti-beta2-glycoprotein I testing: report from the 13th International Congress on Antiphospholipid Antibodies. Arthritis Rheum. 2012;64(1):1-10.

31. Meijide H, Sciascia S, Sanna G, Khamashta MA, Bertolaccini ML. The clinical relevance of $\operatorname{IgA}$ anticardiolipin and $\operatorname{IgA}$ anti-beta2 glycoprotein I antiphospholipid antibodies: a systematic review. Autoimmun Rev. 2013;12(3):421-425.

32. Cousins L, Pericleous C, Khamashta M, et al. Antibodies to domain I of beta-2-glycoprotein I and IgA antiphospholipid antibodies in patients with 'seronegative' antiphospholipid syndrome. Ann Rheum Dis. 2015;74(1):317-319

33. Murthy V, Willis R, Romay-Penabad Z, et al. Value of isolated IgA anti-beta2 -glycoprotein I positivity in the diagnosis of the antiphospholipid syndrome. Arthritis Rheum. 2013;65(12):3186-3193.

34. Pericleous C, Ferreira I, Borghi O, et al. Measuring IgA Anti-beta2-Glycoprotein I and IgG/IgA Anti-Domain I Antibodies Adds Value to Current Serological Assays for the Antiphospholipid Syndrome. Plos One. 2016;11(6):e0156407.

35. Ruiz-Garcia R, Serrano M, Martinez-Flores JA, et al. Isolated IgA anti- beta2 glycoprotein I antibodies in patients with clinical criteria for antiphospholipid syndrome. J Immunol Res. 2014;2014:704395.

36. Tortosa C, Cabrera-Marante O, Serrano M, et al. Incidence of thromboembolic events in asymptomatic carriers of IgA anti ss2 glycoprotein-I antibodies. PloS One. 2017;12(7): e0178889.

37. Sanmarco M, Boffa MC. Antiphosphatidylethanolamine antibodies and the antiphospholipid syndrome. Lupus. 2009;18 (10):920-923

38. Berard M, Chantome R, Marcelli A, Boffa MC. Antiphosphatidylethanolamine antibodies as the only antiphospholipid antibodies. I. Association with thrombosis and vascular cutaneous diseases. J Rheumatol. 1996;23(8):1369-1374.

39. Sanmarco M, Alessi MC, Harle JR, et al. Antibodies to phosphatidylethanolamine as the only antiphospholipid antibodies found in patients with unexplained thromboses. Thromb Haemost. 2001;85(5):800-805.

40. Sanmarco M, Gayet S, Alessi MC, et al. Antiphosphatidylethanolamine antibodies are associated with an increased odds ratio for thrombosis. A multicenter study with the participation of the European Forum on antiphospholipid antibodies. Thromb Haemost. 2007;97(6):949-954.

41. Gris JC, Quere I, Sanmarco M, et al. Antiphospholipid and antiprotein syndromes in non-thrombotic, non-autoimmune women with unexplained recurrent primary early foetal loss. The Nimes
Obstetricians and Haematologists Study-NOHA. Thromb Haemost. 2000;84(2):228236.

42. Billoir P, Miranda S, Abboud J, et al. [Which place of antiphosphatidylethanolamine antibodies research in seronegative antiphospholipid syndrome suspicion?]. Rev Med Interne. 2019;40(6):351-354.

43. Bertolaccini ML, Amengual $O$, Atsumi T, et al. 'Non-criteria' aPL tests: report of a task force and preconference workshop at the 13th International Congress on Antiphospholipid Antibodies, Galveston, TX, USA, April 2010. Lupus. 2011;20(2):191205.

44. Katsuragawa H, Kanzaki H, Inoue T, et al. Monoclonal antibody against phosphatidylserine inhibits in vitro human trophoblastic hormone production and invasion. Biol Reprod. 1997;56(1):50-58.

45. Yetman DL, Kutteh WH. Antiphospholipid antibody panels and recurrent pregnancy loss: prevalence of anticardiolipin antibodies compared with other antiphospholipid antibodies. Fertil Steril. 1996;66(4):540-546.

46. Jaslow CR, Carney JL, Kutteh WH. Diagnostic factors identified in 1020 women with two versus three or more recurrent pregnancy losses. Fertil Steril. 2010;93(4): 1234-1243.

47. Ortona E, Capozzi A, Colasanti T, et al. Vimentin/cardiolipin complex as a new antigenic target of the antiphospholipid syndrome. Blood. 2010;116(16):2960-2967.

48. Musaelyan A, Lapin S, Nazarov V, et al. Vimentin as antigenic target in autoimmunity: A comprehensive review. Autoimmun Rev. 2018;17(9):926-934.

49. Conti F, Capozzi A, Truglia S, et al. The mosaic of "seronegative" antiphospholipid syndrome. J Immunol Res. 2014;2014: 389601.

50. Truglia S, Capozzi A, Mancuso S, et al. A Monocentric Cohort of Obstetric Seronegative Anti-Phospholipid Syndrome. Front Immunol. 2018;9:1678.

51. Sciascia S, Sanna G, Murru V, et al. Anti-prothrombin (aPT) and anti-phosphatidylserine/prothrombin (aPS/PT) antibodies and the risk of thrombosis in the antiphospholipid syndrome. A systematic review. Thromb Haemost. 2014;111(2):354-364.

52. Vaarala O, Puurunen M, Manttari $M$, et al. Antibodies to prothrombin imply a risk of myocardial infarction in middle-aged men. Thromb Haemost. 1996;75(3):456-459.

53. Atsumi $\mathrm{T}$, leko M, Bertolaccini ML, et al. Association of autoantibodies against the phosphatidylserine-prothrombin complex with manifestations of the antiphospholipid syndrome and with the presence of lupus anticoagulant. Arthritis Rheum. 2000;43(9): 1982-1993.

54. Forastiero R, Martinuzzo M, Pombo G, et al. A prospective study of antibodies to beta2glycoprotein I and prothrombin, and risk of thrombosis. J Thromb Haemost. 2005;3(6): 1231-1238

55. Bizzaro N, Ghirardello A, Zampieri S, et al. Anti-prothrombin antibodies predict thrombosis in patients with systemic lupus erythematosus: a 15-year longitudinal study. J Thromb Haemost. 2007;5(6):1158-1164.

56. Heikal NM, Jaskowski TD, Malmberg E, et al. Laboratory evaluation of anti-phospholipid syndrome: a preliminary prospective study of phosphatidylserine/prothrombin antibodies in an at-risk patient cohort. Clin Exp Immunol. 2015;180(2):218-226.

57. Bertolaccini ML, Atsumi T, Koike T, Hughes GR, Khamashta MA. Antiprothrombin anti- 
bodies detected in two different assay systems. Prevalence and clinical significance in systemic lupus erythematosus. Thromb Haemost. 2005;93(2):289-297.

58. Tonello M, Mattia E, Favaro M, et al. IgG phosphatidylserine/prothrombin antibodies as a risk factor of thrombosis in antiphospholipid antibody carriers. Thromb Res. 2019;177:157-160.

59. Bardin N, Alessi MC, Dignat-George F, et al. Does the anti-prothrombin antibodies measurement provide additional information in patients with thrombosis? Immunobiology. 2007;212(7):557-565.

60. Zigon P, Perdan Pirkmajer K, Tomsic M, et al. Anti-Phosphatidylserine/Prothrombin Antibodies Are Associated with Adverse Pregnancy Outcomes. J Immunol Res. 2015;2015:975704.

61. Ganapati A, Goel R, Kabeerdoss J, et al. Study of clinical utility of antibodies to phosphatidylserine/prothrombin complex in Asian-Indian patients with suspected APS. Clin Rheumatol. 2019;38(2):545-553.

62. Zigon P, Podovsovnik A, Ambrozic A, et al. Added value of non-criteria antiphospholipid antibodies for antiphospholipid syndrome: lessons learned from year-long routine measurements. Clin Rheumatol. 2019;38(2):371-378.

63. Singh NK, Yadav DP, Gupta A, Singh U, Godara M. Role of anti-annexin A5 in pathogenesis of hypercoagulable state in patients with antiphospholipid syndrome. Int J Rheum Dis. 2013;16(3):325-330.

64. de Laat B, Derksen RH, Mackie IJ, et al. Annexin A5 polymorphism (-1C-->T) and the presence of anti-annexin A5 antibodies in the antiphospholipid syndrome. Ann Rheum Dis. 2006;65(11):1468-1472.

65. Wolgast LR, Arslan AA, Wu XX, et al. Reduction of annexin A5 anticoagulant ratio identifies antiphospholipid antibody-positive patients with adverse clinical outcomes. J Thromb Haemost. 2017;15(7):1412-1421.

66. Scholz P, Auler M, Brachvogel B, et al Detection of multiple annexin autoantibodies in a patient with recurrent miscarriages, fulminant stroke and seronegative antiphospholipid syndrome. Biochem Med (Zagreb). 2016;26(2):272-278.

67. Sciascia S, Cosseddu D, Montaruli B Kuzenko A, Bertero MT. Risk Scale for the diagnosis of antiphospholipid syndrome. Ann Rheum Dis. 2011;70(8):1517-1518.

68. Otomo K, Atsumi T, Amengual $\mathrm{O}$, et al Efficacy of the antiphospholipid score for the diagnosis of antiphospholipid syndrome and its predictive value for thrombotic events. Arthritis Rheum. 2012;64(2):504512.

69. Oku K, Amengual O, Atsumi T. Antiphospholipid scoring: significance in diagnosis and prognosis. Lupus. 2014;23 (12):1269-1272

70. Sciascia S, Sanna G, Murru V, et al. GAPSS: the Global Anti-Phospholipid Syndrome Score. Rheumatology. 2013;52(8):13971403.

71. Ruiz-Irastorza G, Cuadrado MJ, RuizArruza I, et al. Evidence-based recommendations for the prevention and long-term management of thrombosis in antiphospholipid antibody-positive patients: report of a task force at the 13th International Congress on antiphospholipid antibodies. Lupus. 2011;20(2):206-218

72. Tektonidou MG, Andreoli L, Limper M, et al. EULAR recommendations for the management of antiphospholipid syndrome in adults. Ann Rheum Dis. 2019;78(10):12961304.

73. Bala MM, Paszek E, Lesniak W, et al. Antiplatelet and anticoagulant agents for primary prevention of thrombosis in individuals with antiphospholipid antibodies. Cochrane Database Syst Rev. 2018;7: CD012534.

74. Guyatt GH, Akl EA, Crowther M, et al. Executive summary: Antithrombotic Therapy and Prevention of Thrombosis, 9th ed: American College of Chest Physicians Evidence-Based Clinical Practice Guidelines. Chest. 2012;141(2 Suppl):7S-47S

75. Crowther MA, Ginsberg JS, Julian J, et al. A comparison of two intensities of warfarin for the prevention of recurrent thrombosis in patients with the antiphospholipid antibody syndrome. N Engl J Med. 2003;349 (12):1133-1138

76. Keeling D, Mackie I, Moore GW, et al. Guidelines on the investigation and management of antiphospholipid syndrome. Br Haematol. 2012;157(1):47-58

77. Cohen $H$, Efthymiou M, Isenberg DA. Use of direct oral anticoagulants in antiphospholipid syndrome. J Thromb Haemost. 2018;16(6):1028-1039.

78. Pengo V, Denas G, Zoppellaro G, et al Rivaroxaban vs warfarin in high-risk patients with antiphospholipid syndrome. Blood. 2018:132(13):1365-1371.

79. Goldhaber SZ, Eriksson H, Kakkar A, et al. Efficacy of dabigatran versus warfarin in patients with acute venous thromboembolism in the presence of thrombophilia: Findings from RE-COVER(R), RE-COVER II and RE-MEDY. Vasc Med. 2016;21(6):506514.

80. Woller SC, Stevens SM, Kaplan DA, et al Apixaban for the Secondary Prevention of Thrombosis Among Patients With Antiphospholipid Syndrome: Study Rationale and Design (ASTRO-APS). Clin Appl Thromb Hemost. 2016;22(3):239-247.

81. Canaud G, Bienaime F, Tabarin F, et al Inhibition of the MTORC pathway in the antiphospholipid syndrome. N Engl J Med. 2014;371(4):303-312.

82. Erkan D, Aguiar CL, Andrade D, et al. 14th International Congress on Antiphospholipid Antibodies: task force report on antiphospholipid syndrome treatment trends. Autoimmun Rev. 2014;13(6):685-696.

83. Kumar S, Papalardo E, Sunkureddi P, et al Isolated elevation of IgA anti-beta2glycoprotein I antibodies with manifestations of antiphospholipid syndrome: a case series of five patients. Lupus. 2009;18(11):1011-1014

84. Billoir P, Miranda S, Abboud J, et al. [Which place of antiphosphatidylethanolamine antibodies research in seronegative antiphospholipid syndrome suspicion?]. Rev Med Interne. 2019;40(6):351-354 